\title{
Building BTO System in the Sanitary Materials Manufacturer with the Utilization of the High Accuracy Forecasting
}

\author{
Hirotake Yamashita \\ ${ }^{1}$ College of Business Administration and Information \\ Science, Chubu University, 1200 Matsumoto-cho Kasugai, \\ Aichi,487-8501, Japan
}

\author{
Kazuhiro Takeyasu \\ ${ }^{2}$ College of Business Administration, Tokoha University, \\ 325 Oobuchi, Fuji City, Shizuoka, 417-0801, Japan
}

\begin{abstract}
In recent years, BTO (Build to Order) system is prevailing. It pursues short lead time, minimum stocks, and thereby minimum cost. But the high accuracy demand forecasting is inevitable for the parts manufacturers. In this paper, well organized BTO system in the sanitary materials manufacturer is seek with the aid of high accuracy demand forecasting, which is newly developed by us. Focusing that the equation of ESM is equivalent to $(1,1)$ order ARMA model equation, a new method of estimation of smoothing constant in ESM was derived. Trend removal method was also devised. AR model is also used for forecasting. After removing trend, AR model is utilized and forecasting is executed. Better one in the forecasting accuracy between them was chosen for the final forecasting.
\end{abstract}

Thus, we could obtain the high accuracy demand forecasting. These methods are examined by the data of sanitary materials manufacturer and the BTO system is newly built by utilizing this method. Further development of this system should be performed hereafter.

Keywords-BTO; forecasting; lead time; stock; sanitary materials; AR model

\section{INTRODUCTION}

Supply Chain Management has developed in various aspects. In recent years, BTO (Build to Order) is becoming a big trend. BTO is a system that manufacturers produce goods after they take the order from the customers. The manufacturers can handle the order with the stockless production system. The success story of BTO is found in Dell, where the direct sales to customers have been built. It has the advantage that it has low risk of holding stocks and it can make flexible manufacturing and it can also cope with the customers detailed requests. It is called "Mass Customization" because it has realized the low cost production with mass production and enabled to cope with the customers' requests at the same time. Stockless production with minimum lead time bears a good cost benefit but it has a lot of problems and issues. Even if manufacturer takes a BTO system, parts suppliers must have certain stocks in order to meet the request of manufacturer who gather parts and make assembling. Minimum stocks including parts manufacturer should be pursued. Otherwise, the system would not work long.
Reviewing past papers, there are many researches made on this. G. Parry and A. Graves (2008)[1] and D. M. Anderson (2004)[2] made a versatile overview on BTO. G. Parry and A. Graves (2008) especially focused on the possibility of BTO for automobile manufacturing. Fukushima et al. (2006)[3], Fukushima et al. (2007)[4] made a research on Dell China at Amoy and detailed production scheme including suppliers was analyzed.

In this paper, we are trying to build at the sanitary materials manufacturer, which has a particular circumstance of suppliers and users. Therefore it would be a rare and precious research. One of the most biggest hazard point is how to make correct forecasting. Stock level including parts manufacturers depends much on this.

We proposed a new method of estimation of smoothing constant in ESM before[5]. Focusing that the equation of ESM is equivalent to $(1,1)$ order ARMA model equation, a new method of estimation of smoothing constant in ESM was derived. Trend removal method was also devised. In making forecast such as production data, trend removing method is devised. Trend removing by the combination of linear and 2 nd order non-linear function and 3rd order non-linear function is executed to the data of sanitary materials manufacturer. The weights for these functions are set 0.5 for two patterns at first and then varied by 0.01 increment for three patterns and optimal weights are searched. For the comparison, monthly trend is removed after that. Theoretical solution of smoothing constant of ESM is calculated for both of the monthly trend removing data and the non-monthly trend removing data. Then forecasting is executed on these data. AR model is also used for forecasting. After removing trend, AR model is utilized and forecasting is executed. Better one in the forecasting accuracy between them was chosen for the final forecasting.

In this paper, utilizing above stated method, BTO system for the sanitary materials manufacturer is newly built.

The rest of the paper is organized as follows. In section 2, current status and issues of sanitary materials manufacturer are stated. Section 3 through 6 are the description of forecasting method. In section 3, ESM is stated by ARMA model and estimation method of smoothing constant is derived using 
ARMA model identification. The combination of linear and non-linear function is introduced for trend removing in section 4. The Monthly Ratio is referred in section 5. AR model is described in section 6. Forecasting is executed in section 7 , and estimation accuracy is examined. In section 8 , building BTO system is stated, which is followed by the conclusion of section 9.

\section{CURRENT STATUS AND ISSUES OF SANITARY MATERIALS MANUFACTURER}

\section{A. Outline of Sanitary Materials Manufacturer}

Outline of Sanitary Materials Manufacturer (Company O) is as follows.

/ Established: 1936

/ Business Domain: Production and sales of Sanitary Materials

/ Business Department and Product:

\section{A. Medical Department}

absorbent gauze, absorbent cotton, products for maternity clinic, products for gynecological department, products for surgery

\section{B. Consumer Department}

hygiene cotton, products for nursing and nursing care

/ Base:

A. Headquarter

Nagoya

B. Sales Base

10 places in Japan

Shanghai

C. Factory

3 factories in Japan

Indonesia (Affiliated)

/ Number of Product Items: 3943 (Real Moving one: about 1600)

/ Lead Time for the Production:

A. Sterilized Product: 10 12 days

B. Non Sterilized Product: 5 7 days

I ABC Analysis in the Sales Amount (September 2011 through August 2012)

Total items: 3943

Rank A items (Upper 70\%) 577

Rank B items (Upper 90\%) 727

Rank C items

2639

\section{B. Problems and Issues}

Under the severe competition with other companies, Company $\mathrm{O}$ has took every means in order to reduce cost. The methods they took are as follows.

/ Increase Sales Base so as to respond to the customers' needs

/ Increase distribution center

/ Increase purchase from abroad

/ Build factory in abroad

Partially, these made contribution of cost reduction, but caused disadvantage of the increase of stocks and prolonged lead time.

Imported parts from abroad consist of nearly $20 \%$ and these take more than one month's lead time, which causes increase of stocks. Each department made every effort in each department and that did not make any optimization in the total.

There are several means for the total optimization. Among them, correct forecasting is inevitable in the total supply chain management.

From now on, the newly developed forecasting method by us is stated in section 3 through 6 and forecasting is executed in section 7 .

This time, we set Rank A items (Share is $15 \%$ in the total items) as important items to be controlled and make forecasting for them. The base data for forecasting is each sales amount of September 2009 through August 2012.

\section{DESCRIPTION OF ESM USING ARMA MODEL}

\section{A. ESM and ARMA mode}

In ESM, forecasting at time $t+1$ is stated in the following equation.

$$
\begin{aligned}
\hat{x}_{t+1} & =\hat{x}_{t}+\alpha\left(x_{t}-\hat{x}_{t}\right) \\
& =\alpha x_{t}+(1-\alpha) \hat{x}_{t}
\end{aligned}
$$

Here,

$$
\begin{aligned}
\hat{x}_{t+1} & : \text { forecasting at } t+1 \\
x_{t}: & \text { realized value at } t \\
& \alpha: \text { smoothing constant }(0<\alpha<1)
\end{aligned}
$$

(2) is re-stated as

$$
\hat{x}_{t+1}=\sum_{l=0}^{\infty} \alpha(1-\alpha)^{l} x_{t-l}
$$

By the way, we consider the following $(1,1)$ order ARMA model.

$$
x_{t}-x_{t-1}=e_{t}-\beta e_{t-1}
$$

Generally, $(p, q)$ order ARMA model is stated as 


$$
x_{t}+\sum_{i=1}^{p} a_{i} x_{t-i}=e_{t}+\sum_{j=1}^{q} b_{j} e_{t-j}
$$

Here,

$\left\{x_{t}\right\}$ : Sample process of Stationary Ergodic Gaussian Process $x(t) t=1,2, \cdots, N, \cdots$

$\left\{e_{t}\right\}$ : Gaussian White Noise with 0 mean $\sigma_{e}^{2}$ variance

MA process in (5) is supposed to satisfy convertibility condition.

Utilizing the relation that

$$
E\left[e_{t} \mid e_{t-1}, e_{t-2}, \cdots\right]=0
$$

we get the following equation from (4).

$$
\hat{x}=x_{t-1}-\beta e_{t-1}
$$

Operating this scheme on $t+1$, we finally get

$$
\begin{aligned}
& \hat{x}_{t+1}=\hat{x}_{t}+(1-\beta) e_{t} \\
& =\hat{x}_{t}+(1-\beta)\left(x_{t}-\hat{x}_{t}\right)
\end{aligned}
$$

If we set $1-\beta=\alpha$, the above equation is the same with (1), i.e., equation of ESM is equivalent to $(1,1)$ order ARMA model, or is said to be $(0,1,1)$ order ARIMA model because 1 st order AR parameter is -1 .

\section{B. Estimation of Smoothing Constant utilizing System \\ Identification of ARMA model}

Comparing with (4) and (5), we obtain.

$$
\left\{\begin{array}{l}
a_{1}=-1 \\
b_{1}=-\beta=\alpha-1
\end{array}\right.
$$

From (1), (7),

$$
\alpha=1-\beta
$$

Therefore, we get

$$
\left\{\begin{array}{l}
a_{1}=-1 \\
b_{1}=-\beta=\alpha-1
\end{array}\right.
$$

From above, we can get estimation of smoothing constant after we identify the parameter of MA part of ARMA model. But, generally MA part of ARMA model become non-linear equations which are described below.

Let (5) be

$$
\tilde{x}_{t}=x_{t}+\sum_{i=1}^{p} a_{i} x_{t-i}
$$

$$
\tilde{x}_{t}=e_{t}+\sum_{j=1}^{q} b_{j} e_{t-j}
$$

We express the autocorrelation function of $\tilde{x}_{t}$ as $\tilde{r}_{k}$ and from (9), (10), we get the following non-linear equations which are well known.

$$
\begin{aligned}
& \tilde{r}_{k}= \begin{cases}\sigma_{e}^{2} \sum_{j=0}^{q-k} b_{j} b_{k+j} \quad(k \leq q) & (k \geq q+1) \\
0 & (k \geq q\end{cases} \\
& \tilde{r}_{0}=\sigma_{e}^{2} \sum_{j=0}^{q} b_{j}^{2}
\end{aligned}
$$

For these equations, recursive algorithm has been developed. In this paper, parameter to be estimated is only $b_{1}$, so it can be solved in the following way.

From (4) (5) (8) (11), we get

$$
\begin{aligned}
& q=1 \\
& a_{1}=-1 \\
& b_{1}=-\beta=\alpha-1 \\
& \tilde{r}_{0}=\left(1+b_{1}^{2}\right) \sigma_{e}^{2} \\
& \tilde{r}_{1}=b_{1} \sigma_{e}^{2}
\end{aligned}
$$

If we set

$$
\rho_{k}=\frac{\tilde{r}_{k}}{\tilde{r}_{0}}
$$

the following equation is derived.

$$
\rho_{1}=\frac{b_{1}}{1+b_{1}^{2}}
$$

We can get $b_{1}$ as follows.

$$
b_{1}=\frac{1 \pm \sqrt{1-4 \rho_{1}^{2}}}{2 \rho_{1}}
$$

In order to have real roots, $\rho_{1}$ must satisfy

$$
\left|\rho_{1}\right| \leq \frac{1}{2}
$$

From invertibility condition, $b_{1}$ must satisfy

$$
\left|b_{1}\right|<1
$$

From (14), using the next relation, 
(16) always holds.

$$
\begin{aligned}
& \left(1-b_{1}\right)^{2} \geq 0 \\
& \left(1+b_{1}\right)^{2} \geq 0
\end{aligned}
$$

As

$$
\alpha=b_{1}+1
$$

$b_{1}$ is within the range of

$$
-1<b_{1}<0
$$

Finally we get

$$
\left.\begin{array}{l}
b_{1}=\frac{1-\sqrt{1-4 \rho_{1}^{2}}}{2 \rho_{1}} \\
\alpha=\frac{1+2 \rho_{1}-\sqrt{1-4 \rho_{1}^{2}}}{2 \rho_{1}}
\end{array}\right\}
$$

which satisfy above condition. Thus we can get a theoretical solution by a simple way.

Focusing that the equation of ESM is equivalent to $(1,1)$ order ARMA model equation, we can estimate smoothing constant after estimating ARMA model parameter.

It can be estimated only by calculating 0th and 1st order autocorrelation function

\section{TRend Removal Method}

As a trend removal method, we describe the combination of linear and non-linear function.

[1] Linear function

We set:

$$
y=a_{1} x+b_{1}
$$

as a linear function.

[2] Non-linear function

We set:

$$
\begin{gathered}
y=a_{2} x^{2}+b_{2} x+c_{2} \\
y=a_{3} x^{3}+b_{3} x^{2}+c_{3} x+d_{3}
\end{gathered}
$$

as a $2^{\text {nd }}$ and a $3^{\text {rd }}$ order non-linear function.

[3] The combination of linear and non-linear function

We set:

$$
\begin{gathered}
y=\alpha_{1}\left(a_{1} x+b_{1}\right)+\alpha_{2}\left(a_{2} x^{2}+b_{2} x+c_{2}\right) \\
y=\beta_{1}\left(a_{1} x+b_{1}\right)+\beta_{2}\left(a_{3} x^{3}+b_{3} x^{2}+c_{3} x+d_{3}\right) \\
y=\gamma_{1}\left(a_{1} x+b_{1}\right)+\gamma_{2}\left(a_{2} x^{2}+b_{2} x+c_{2}\right) \\
+\gamma_{3}\left(a_{3} x^{3}+b_{3} x^{2}+c_{3} x+d_{3}\right)
\end{gathered}
$$

as a combination of linear and 2 nd order non-linear and 3 rd order non-linear function. Here, , , . Trend is removed by dividing the original data by (21),(22) and (23). Comparative discussion concerning (21), (22) and (23) is described in section 5 .

\section{Monthly Ratio}

For example, if there is the monthly data of $\mathrm{L}$ years as stated bellow:

$$
\left\{x_{i j}\right\}(i=1, \cdots, L)(j=1, \cdots, 12)
$$

where, $x_{i j} \in R$ in which $j$ means month and $i$ means year and $x_{i j}$ is a shipping data of $\mathrm{i}$-th year, $\mathrm{j}$-th month, then, monthly ratio $\tilde{x}_{j}(j=1, \cdots, 12)$ is calculated as follows.

$$
\tilde{x}_{j}=\frac{\frac{1}{L} \sum_{i=1}^{L} x_{i j}}{\frac{1}{L} \cdot \frac{1}{12} \sum_{i=1}^{L} \sum_{j=1}^{12} x_{i j}}
$$

Monthly trend is removed by dividing the data by (24). Numerical examples both of the monthly trend removal case and the non-removal case are discussed in section 7.

\section{AR Model by Using Delay OPERATOR}

$p$ - th order AR model is stated as follows.

$$
x_{t}+a_{1} x_{t-1}+\cdots+a_{p} x_{t-p}=e_{t}
$$

\section{ForecAsting THE SHIPPING Data OF MANUfactureR}

\section{A. Analysis Procedure}

The shipping data of sanitary materials manufacture for 4 cases from September 2009 through August 2012 are analyzed. Contents of these are as follows.

Case 1: Sum data of all Rank A items

Case 2: Product A (absorbent cotton)

Case 3: Product B (Set product for birth)

Case 4: Product C (Material of set product for birth) 
Analysis procedure is as follows. There are 36 monthly data for each case. We use 24 data (1st to 24th) and remove trend by the method stated in section 4 . Then we calculate monthly ratio by the method stated in section 5. After removing monthly trend, the method stated in section 3 is applied and Exponential Smoothing Constant with minimum variance of forecasting error is estimated. Then 1 step forecast is executed. Thus, data is shifted to 2 nd to 25 th and the forecast for 26th data is executed consecutively, which finally reaches forecast of 36th data. To examine the accuracy of forecasting, variance of forecasting error is calculated for the data of 25th to 36th data. Final forecasting data is obtained by multiplying monthly ratio and trend.

Forecasting error is expressed as:

$$
\begin{gathered}
\varepsilon_{i}=\hat{x}_{i}-x_{i} \\
\bar{\varepsilon}=\frac{1}{N} \sum_{i=1}^{N} \varepsilon_{i}
\end{gathered}
$$

Variance of forecasting error is calculated by:

$$
\sigma_{\varepsilon}^{2}=\frac{1}{N-1} \sum_{i=1}^{N}\left(\varepsilon_{i}-\bar{\varepsilon}\right)^{2}
$$

These schemes are similarly adapted to AR model stated in section 6. We set Forecasting Accuracy Ratio (FAR) as follows.

$$
F A R=\left(1-\sum_{i=1}^{N} \frac{\left|\hat{x}_{i}-x_{i}\right|}{x_{i}}\right) \times 100(\%)
$$

The method that has better PAR value is adopted between them.

\section{B. Forecasting Results}

Forecasting results for 4 cases are exhibited in Figure 1 through Figure 4.

Forecasting results for 4 cases are exhibited in Figure 1 through Figure 4.

We can observe that each case has a very good forecasting accuracy.

We have confirmed the FAR for all the Rank A items and materials of set product for birth in Rank A. Summary of them is exhibited at Table 2 .

Summary of them is exhibited at Table 2.

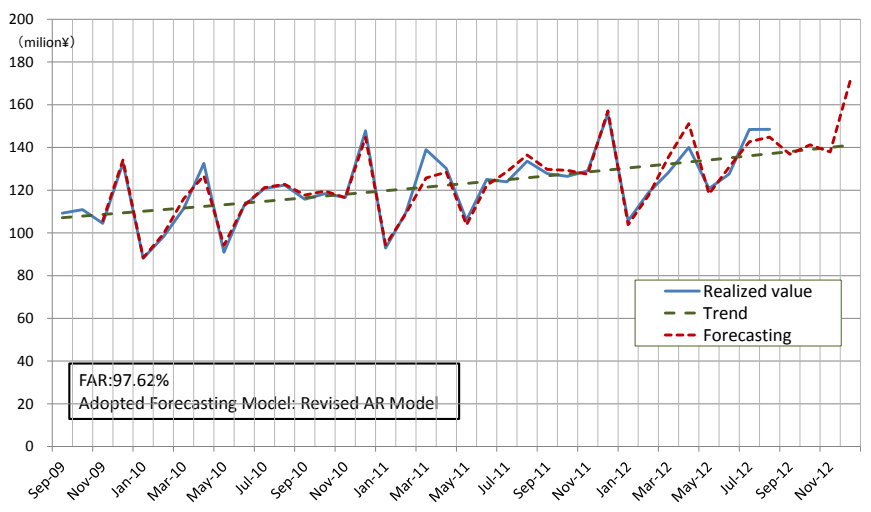

Fig. 1. Forecasting result for Case 1

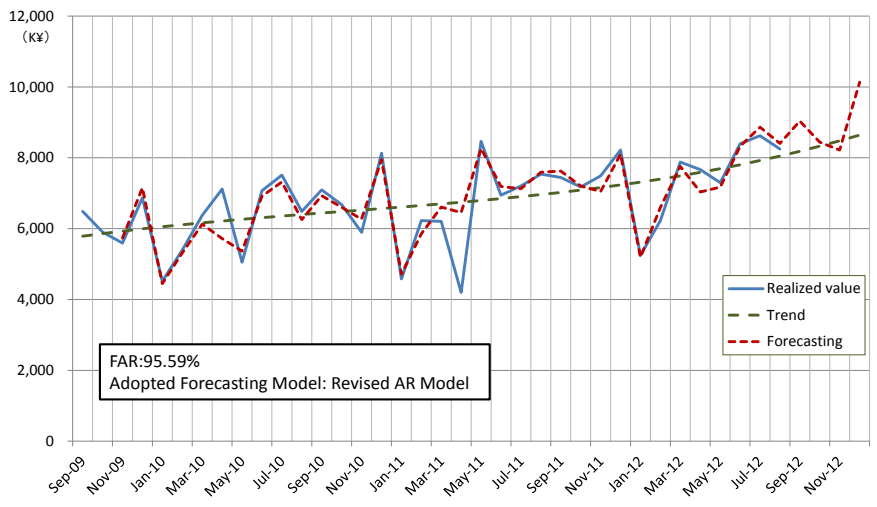

Fig. 2. Forecasting result for Case 2

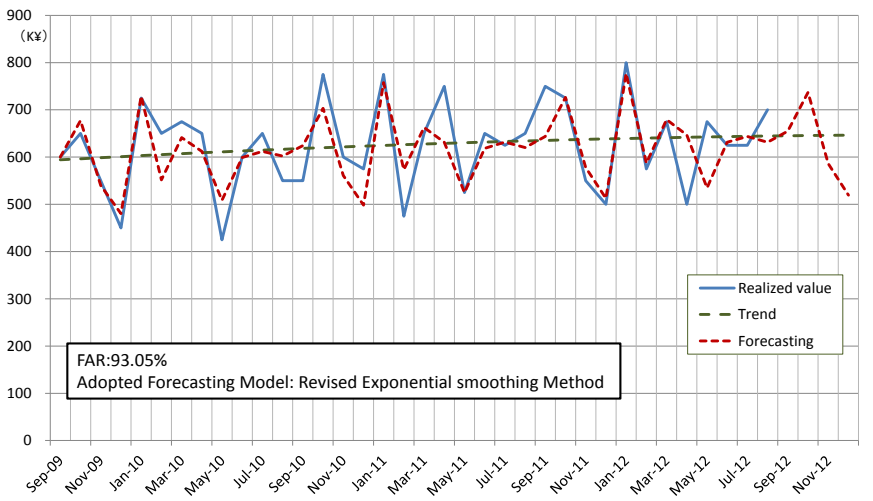

Fig. 3. Forecasting result for Case 3

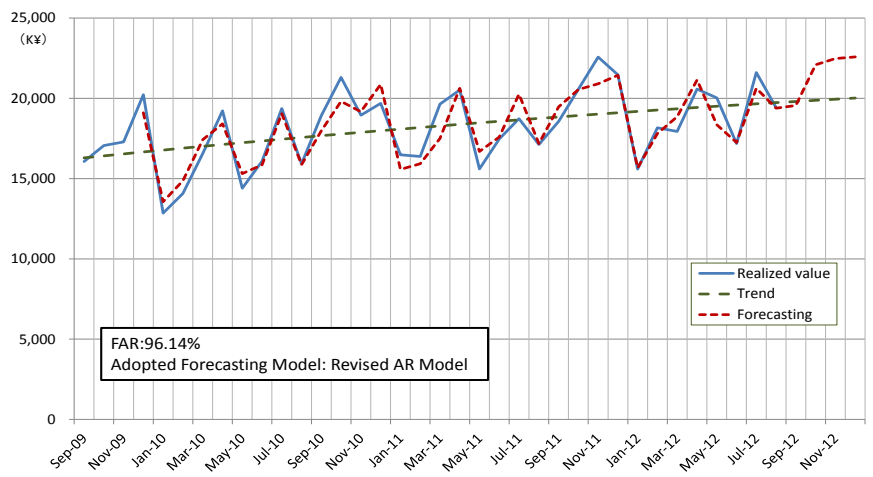

Fig. 4. Forecasting result for Case 4 


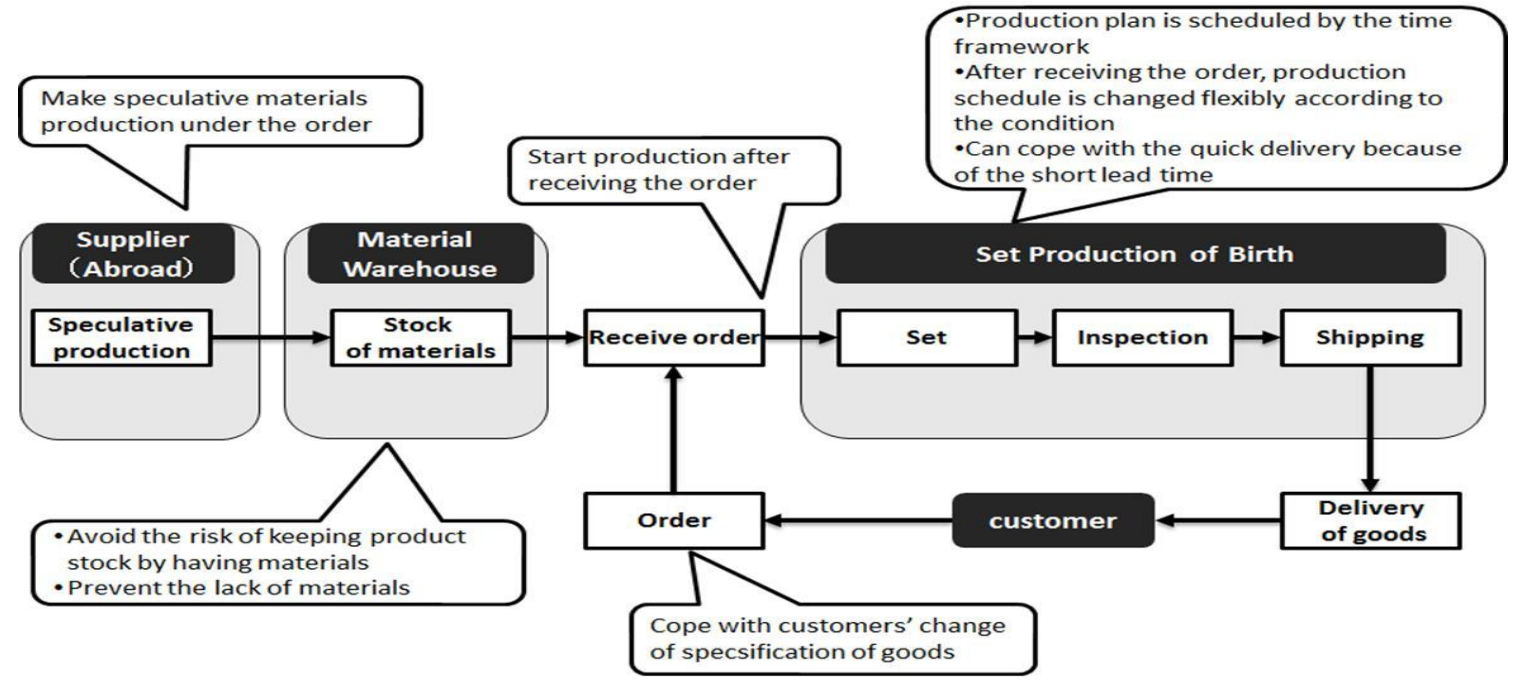

Fig. 5. The Scheme of BTO for the Set Production of Birth

TABLE I. THEIR SUMMARY IS EXHIBITED IN TABLE 1

\begin{tabular}{|c|c|c|}
\hline & Selected Model & FAR (\%) \\
\hline Case1 & Revised AR Mode & 97.62 \\
\hline Case2 & $\begin{array}{c}\text { Revised Exponential Smoothing } \\
\text { Method }\end{array}$ & 95.59 \\
\hline Case3 & Revised AR Mode & 93.05 \\
\hline Case4 & Revised AR Mode & 96.14 \\
\hline
\end{tabular}

TABLE II. FAR For ALl THE RANK A ITEMS

\begin{tabular}{|c|c|c|c|c|c|}
\hline & \multirow{2}{*}{$\begin{array}{c}\text { Number } \\
\text { of items }\end{array}$} & \multicolumn{3}{|c|}{ Distribution (FAR) (\%) } & Mode \\
\cline { 3 - 5 } & $\sim 85 \%$ & $\sim 80 \%$ & $\sim 75 \%$ & \\
\hline $\begin{array}{c}\text { All the Rank } \\
\text { A items }\end{array}$ & 577 & 70.2 & 86.5 & 92.7 & 90.0 \\
\hline $\begin{array}{c}\text { Materials of } \\
\text { Set Product } \\
\text { for Birth }\end{array}$ & 124 & 97.6 & 97.6 & 98.4 & 96.0 \\
\hline
\end{tabular}

The mode is $90 \%$ for all the Rank A items.

We take up materials of set product for birth in Rank A and the mode is $96 \%$. These are sufficiently enough to utilize these forecasting methods to the actual daily business.

\section{BuILding The BTO System For the Set PRoduction OF BIRTH}

The construction of materials for the set product of birth differs by customer. Therefore procurement becomes difficult. Exquisite forecasting of materials of set product for birth enables procurement to be a stable and correct one, which would contribute to keep the appropriate stock level. From this condition, building the BTO system for the set product of birth is a good way to this set product. In Figure 5, the scheme of BTO system for the set product of birth is exhibited.

Factory does not have product stocks basically. Planner makes order sheet based upon the forecasting results of materials, and he announce unofficially to the suppliers (Abroad, for example, Indonesia) for 3 through 4 months' preliminary order quantities. Suppliers make production under this preliminary order. Thus, it helps the shortening of procurement lead time and lack of materials. After receiving the order from the customer, they soon start production and make delivery after it is completed. The operation is mainly consisted by the hand work of making set, therefore replacing the product with other products can easily be done.

By shortening the lead time, they can cope with the request of customers flexibly. Improvement of cash flow is also achieved by decreasing stock level.

Now, we summarize the advantage of forecasting in this company.

/ Exquisite production planning can be achieved by utilizing the result of precise forecasting. Demand forecasting is the basis and the starting point of Supply Chain Management.

/ Exquisite preliminary order can be built, which causes less stocks and shorter lead time in suppliers.

/ Smooth BTO is established with short lead time and stockless production by co-operating with suppliers, which will derive win-win relationship among maker and suppliers..

\section{CONCLUSION}

In recent years, BTO (Build to Order) system is prevailing. It pursues short lead time, minimum stocks, and thereby minimum cost. But the high accuracy demand forecasting is inevitable for the parts manufacturers.

In this paper, well organized BTO system in the sanitary materials manufacturer is seek with the aid of high accuracy demand forecasting, which is newly developed by us. Focusing that the equation of ESM is equivalent to $(1,1)$ order ARMA model equation, a new method of estimation of smoothing constant in ESM was derived. Trend removal method was also devised. AR model is also used for forecasting. After removing trend, AR model is utilized and forecasting is executed. Better one in the forecasting accuracy between them was chosen for the final forecasting. Thus, we could obtain the high accuracy demand forecasting. These methods are examined by the data of sanitary materials manufacturer and the BTO system is newly built by utilizing this method. Smooth BTO is established with short lead time 
and stockless production by co-operating with suppliers, which will derive win-win relationship among maker and suppliers. By shortening the lead time, they can cope with the request of customers flexibly. Improvement of cash flow is also achieved by decreasing stock level..

\section{FUTURE WORKS}

Future works of this paper are as follows.

/ To examine the advantage in the performed case

/ To further develop the BTO system in the performed case

/ To Extend the BTO system in various cases

Further development of this system should be performed hereafter. In the end, we appreciate Mr. Norio Funato for his helpful support of our study.

\section{REFERENCES}

[1] G. Parry and A. Graves Editors, Build To Order: The Road to the 5-Day Car, Springer, 2008.

[2] D. M. Anderson, Build-to-Order \& Mass Customization, CIM Press, 2004.

[3] Fukushima, Kazunobu / Kohmura, Toshitaka / Oshima, Taku / Zhang, Jixun / Kiuchi, Masamitsu, "Developing Supply Chains of Japanese Manufacturers in China", Josai management review, Vol.2,No.1,pp.97$110,2006$.

[4] Fukushima, Kazunobu / Kohmura, Toshitaka / Oshima, Taku / Zhang, Jixun / Kiuchi, Masamitsu, "Developing Supply Chains of Japanese Manufacturers in China (Part 2)", Josai management review, Vol.3, No.1, pp.47-60, 2007.

[5] Kazuhiro Takeyasu and Keiko Nagata, "Estimation of Smoothing Constant of Minimum Variance with Optimal Parameters of Weight", International Journal of Computational Science Vol.4,No.5, pp. 411425,2010 